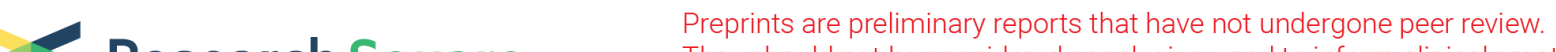 Research Square
Thr ey should not be considered conclusive, used to inform clinical practice,
of the the media as validated information.
}

\section{Catastrophic Carpal Fractures in Racehorses during Traditional Competitions: Assessing Mechanobiology using Multibody Modelling and Comparative Analysis}

\section{Eleonora Pagliara}

University of Turin

Alvise Pasinato

University of Turin

Alberto Valazza

University of Turin

Federica Cantatore

University of Turin

Mara Terzini

Polytechnic University of Turin

Giovanni Putame

Polytechnic University of Turin

Annapaola Parrilli

Empa - Swiss Federal Laboratories for Materials Science and Technology

Maria Sartori

IRCCS-Istituto Ortopedico Rizzoli, Complex Structure of Surgical Sciences and Technologies

Milena Fini

IRCCS-Istituto Ortopedico Rizzoli, Complex Structure of Surgical Sciences and Technologies

\section{Elisabetta Zanetti}

University of Perugia

Andrea Bertuglia ( $\square$ andrea.bertuglia@unito.it )

University of Turin

\section{Research Article}

Keywords: horse, traditional race, carpal fracture, micro-computed tomographic $(\mu \mathrm{CT})$ analysis, inverse dynamic analysis, multibody dynamic simulation, in silico modelling, computed modelling

Posted Date: March 15th, 2021 
DOl: https://doi.org/10.21203/rs.3.rs-289973/v1

License: (c) (1) This work is licensed under a Creative Commons Attribution 4.0 International License. Read Full License 


\section{Abstract}

Background: City circuit competitions (Palio) in Italy are traditional horse race held in the heart of some cities centre once a year. The prevalence of accidents during these competitions is only anecdotally reported; however there is a diffuse perception that these events adversely affect racehorse industry and impact on equine welfare. The aim of the study is to understand the basic physiopathology of catastrophic fracture of the carpus in a 7-y old thoroughbred euthanized following catastrophic fracture of the left carpus during a traditional horse race, comparing the localization and the magnitude of the contact forces at the level of the affected joint, obtained in simulated conditions with the macro- and microscopic structural damages.

Methods: A retrospective analysis of a thoroughbred racehorse galloping at high speeds on an urban racetrack conditions was set up. Computational modelling of the carpal joint was generated using a multibody code for dynamics simulation, considering the circuit design, the speed of the animal, and the surface characteristic. The results were compared to the findings observed during computed tomography, micro-computed tomography and histological evaluation following hematoxylin and eosin and safranin0 fast green staining.

Results: The articular surfaces of the radial, intermediate and ulnar facet of the radius together with the proximal articular surface of small carpal bones exhibited diffuse wear lines, erosions of the articular cartilage and subchondral bone exposure. The fracture line along the radial carpal bone initiating the injury does not travel in a region with bone sclerosis. In the computational model, the peak force for the contact between the radius and the radial carpal bone has a value of $6880 \mathrm{~N}$.

Conclusions: This study highlight how during traditional racing circuits it develops elevate impact forces at the level of contact surfaces of the carpal joint, due to effect of speed and curve, causing catastrophic bone fracture and hyaline cartilage breakdown in the absence of pre-existing pathology.

\section{Background}

In large cursorial mammals, like horses at gallop, vertical contact forces are transmitted across diarthrodial joints of the forelimb, when the carpus is loaded in full extension at gallop. Carpus in horses is an high-motion joint transferring all the loading generated at high-speed from the radius to the cannon bone. The medial aspect of the carpal joints is subjected to a higher mechanical stress when the joint is closed, due to the anatomical arrangement of the cuboid bones one above the other. The antebrachiocarpal joint is a hinge-like joint, where the full contact of the articular surfaces realizes at the peak of the stance phase of the stride. An elevated contact pressures between the opposing surfaces is registered at the medial aspect of the third carpal bone with increasing loading [1].

The articular cartilage, a highly hydrated and specialized tissue, acts to accommodate mechanical load during heavy contact between opposite joint surfaces. In the range of physiological loading, articular cartilage shows a viscoelastic behaviour. The interstitial fluid is gently squeezed out from the cartilage 
and this mechanism is designed to resist elevated compressive stress, saving the bone from mechanical failure [2]. Repetitive supra-physiological impact is recognized to produce chondrocyte necrosis and cell apoptosis in the cartilage tissue [3], triggering the subsequent degenerative process. The progressive degeneration of hyaline cartilage changes dynamic behaviour, increasing matrix permeability to the interstitial fluid and the risk of bone failure. Catastrophic carpal fractures in racehorses are considered a final event in those areas where repeated cyclic load produced elevated stress in the bone, in presence of progressive degeneration of the hyaline cartilage [4]. Adaptive changes of the subchondral bone (SCB) are well documented in racehorses in training [5], as a part of unique maladaptive process of bone and hyaline cartilage $[4,6]$. The role of a single acute joint trauma to produce a structural damage in the hyaline cartilage and in the bone is still debated.

Traditional races are historical events that take place in many Italian mediaeval cities, where the main square is prepared and covered with a deep layer of tuff clay to host a competition once a year. The equine welfare is a significative concern during those events, due to dramatic injuries experienced by many horses. An elevated number of catastrophic fractures during traditional races have been only anecdotally reported, and specific data on the incidence of carpal fractures during those races is currently unavailable.

Computational modelling represents a viable alternative to experimentation in the field of biomechanics. Especially, numerical multibody models have proven to be a valuable tool for addressing open issues related to the musculoskeletal system as well as improving the understanding of complex anatomical structures, like those constituting arthrodial joints. By exploiting these models, kinematics and dynamics quantities that are difficult, or even impossible to be directly measured experimentally (e.g., intra-articular contact forces), can be estimated to enable, for instance, the identification of detrimental biomechanical conditions, thus allowing the prediction of potential injuries $[7,8]$. In order to be implemented, such computational models need of input data which can be derived from imaging (e.g., computed tomography files), literature, experimental or measurements.

The aim of this study is to use multibody analysis, performed through computer simulations, to understand the basic physiopathology of catastrophic fracture of the equine carpal joint under loading conditions of a racing scenario. Therefore, the position and magnitude of the intra-articular contact forces, originated at the antebrachio-carpal joint, resulting from multibody simulations and including case-specific race conditions and articular geometries were compared with the macro- and microscopic structural damages observed post-mortem within the joint of a horse. The model could potentially identify the critical element that led to the injury, giving the opportunity to reduce the number of catastrophic injuries during those events. The hypothesis of this study was that the results of the in silico model compared with the findings of the imaging techniques and histological evaluation would have been consistent with an acute injury in a racehorse experienced a catastrophic carpal fracture.

\section{Results}


Radiographic examination revealed a biarticular and moderately displaced fracture of the radial carpal bone $(\mathrm{Cr})$ associated with comminution of the palmaro-proximal aspect of the intermediate carpal bone (Ci) and a comminute displaced open fracture of the proximal portion of the fourth metacarpal bone (Mc4) in the left forelimb (LF).

\section{Computed Tomography (CT)}

Macroscopic morphology of the fracture lines has been defined using two-dimensional (2D) elaboration of the CT scans. The $\mathrm{Cr}$ showed a complete fracture along the coronal plane, and propagating from the antebrachio-carpal to the intercarpal joint, with multiple small fragmentations at the fracture margins. This main fracture line divided the $\mathrm{Cr}$ in 2 pieces; where the dorsal portion was approximately $2 / 3$ of the whole bone volume and the palmar portion correspond to $1 / 3$ of the entire volume of the bone. A second fracture was observed at the middle portion of the Mc4, where the sharp fragments of this bone exposed the fracture line throughout the skin. The middle portion of the Mc4 was dislodged due to the disruption of the inter-metacarpal ligament and it was interpreted as an avulsion fracture due the tremendous force generated in the metacarpal fascia during the accident. The complete series of transverse CT scans from the middle of the radius to the third metacarpal bone are available (Additional file 1).

\section{Pathology}

At the level of the antebrachiocarpal joint the articular surfaces of the radial, intermediate and ulnar facet of the radius exhibited diffuse wear lines, extensive erosions of the articular cartilage and two regions with patchy areas of cartilage erosion and SCB exposure. The ulnar carpal bone $(\mathrm{Cu})$ presented multiple wear lines, whereas the articular surfaces of the $\mathrm{Cr}$ and $\mathrm{Ci}$ exhibited irregular erosions and full thickness destruction of the articular cartilage. Articular surface of the antebrachio-carpal joint was classified as grade 3 and 4, due to the extensive retention of Indian Ink at the level of the hyaline cartilage (Fig. 1). The direction of the wear lines was coincident with the physiologic rotation axis of the joint. The articular surface of the intercarpal joint was classified as grade 1 and 2, due to the limited retention of Indian Ink at the articular surface. At the level of the carpo-metacarpal joint the articular surfaces of the bones presented normal appearance.

\section{Qualitative and quantitative $\mu \mathrm{CT}$ analysis}

$\mu C T$ datasets allow to virtually cut the images along different anatomical planes and to analyse very rapidly the trabecular pattern of SCB and trabecular bone of the distal epiphysis of the radius and the small carpal bones. We used both Dataviewer (Bruker microCT, Belgium) and Image $\mathrm{J}$ software loading the entire datasets as virtual stacks.

Observing the articular surface of the radius using the false colour light-blue attributed to the hyaline cartilage, three regions with a severe focal cartilage lesion were detected at the level of the radial and intermediate articular surface of the radius, confirming the macroscopic observation (Fig. 2). Two lesions were localized on the radial articular facet, one on the dorso-medial border of the bone and the second in 
the dorsolateral aspect of the radial epicondyle. Further cartilage lesion was observed in the middle of the palmar aspect of the intermediate epicondyle.

In SBC and trabecular areas, neither focal porosities nor surface irregularities were detected. Sections along the sagittal plans revealed bone densification over the SCB regions in a limited area at the level of the dorsal aspect of the radial and intermediate facet of the distal radius, and at the dorsal aspect of the $\mathrm{Cr}$ and $\mathrm{Ci}$ (Fig. 3). Those areas of bone sclerosis were considered in the physiologic range for this 7-yearsold TB racehorse with a long racing career. Interestingly, the fracture line along the $\mathrm{Cr}$ does not travel a region with bone sclerosis.

Three volume of interest (VOI) of $15 \mathrm{~mm}$ in size were selected on the distal articular surface of radius, based on the severity of macroscopic aspect of the articular cartilage. The VOI 1 and 2 were located axially to the medial parasagittal ridge, in the medial styloid process of the radius. The VOI 1 was opposite to the fracture line on the $\mathrm{Cr}$, while the $\mathrm{VOI} 3$ is the negative control and is located abaxially to the medial parasagittal ridge, at the level of the intermediate facet of the radius. The VOIs sampled in the distal articular surface of the radius showed marked differences in cartilage thickness (C.Th): in the VOI 1 , it was detected the minimum value of $C$.Th $(76.78 \mu \mathrm{ms})$. The values of the $C$.Th together with the other structural $\mu \mathrm{CT}$ parameters were reported in detail in the corresponding table (Table 1).

Table 1

$\mu \mathrm{CT}$ parameters

Trabecular bone Cartilage

\begin{tabular}{|c|c|c|c|c|c|c|c|c|}
\hline VOI & BV/TV & Tb.Th & Tb.SP & Tb.N & Tb.Pf & SMI & $\mathrm{Da}$ & C.th \\
\hline & $\%$ & $\mu \mathrm{m}$ & $\mu \mathrm{m}$ & & & & & $\mu \mathrm{m}(\min -\max )$ \\
\hline Total & 40.76 & 309.44 & 507.32 & 1.32 & -0.19 & -0.09 & 0.54 & \\
\hline Area 1 & 35.40 & 199.89 & 514.88 & 1.77 & -0.44 & 0.15 & 0.32 & $224.59(76.78-579.90)$ \\
\hline Area 2 & 51.52 & 334.81 & 423.31 & 1.54 & -2.44 & -1.37 & 0.40 & $312.29(99.61-567.29)$ \\
\hline Area 3 & 41.21 & 273.97 & 515.78 & 1.50 & -0.73 & -0.35 & 0.56 & $629.83(319.86-825.79)$ \\
\hline \multicolumn{9}{|c|}{$\begin{array}{l}\text { Table 1. Caption: Quantitative } \mu C T \text { data of selected volume of interest (VOI) from the antebrachio- } \\
\text { carpal joint, taken at the distal radius, as shown in the corresponding illustration. Parameters } \\
\text { measured included bone volume fraction (BV/TV), trabecular thickness (Tb.Th), trabecular spacing } \\
\text { (Tb.Sp) and number (Tb.N), fragmentation index (Tb.Pf), structure model index (SMI), degree of } \\
\text { anisotropy (Da) and cartilage thickness (C.Th). C.Th. has been reported as a mean value and also } \\
\text { with the minimal and maximal value. }\end{array}$} \\
\hline
\end{tabular}

\section{Histopathology}

The cartilage sections stained with safranin-O fast green (SOFG) were assessed using a validated grading system (Fig. 4). The sections stained with hematoxylin and eosin (H\&E) were similarly assessed 
for comparison. Cartilage scores of each section are reported in Table 2. Hyaline cartilage at the level of the radial facet appeared extensively torn.

Table 2

Distal radius cartilage score

\section{Topographic sections of the distal radius \\ Histologic grading score of the HAC}

-Ulnar surface (A),

Grade 0 (i.e. intact cartilage surface and intact cartilage

-Ulnar surface (B), morphology)

-Ulnar surface (C),

-Intermediate surface (D),

-Intermediate surface (E),

-Intermediate surface (F),

-Intermediate surface (G),

-Radial surface (M),

-Radial surface $(\mathrm{N})$

-Radial surface (F)

Grade 2 (i.e. surface discontinuity)

-Radial surface $(H)$,

Grade 4 (i.e. cartilage erosion)

-Radial surface (I),

-Radial surface (0).

-Radial surface (L),

Grade 5 (i.e. cartilage denudation)

-Radial surface (P).

Table 2 caption: Histopathology grading of the hyaline cartilage of the entire surface of the distal radius, according with a modified Mankin score (Mcllwraith, 2010). Corresponding sections are reported in the illustration.

\section{Computation modelling analysis}

From the computational model all three carpal bones $(\mathrm{Cr}, \mathrm{Ci}, \mathrm{Cu})$ resulted in contact with the radius after $18.8 \mathrm{~ms}$ from the beginning of the simulated stride. Contact forces present a peak soon after the trailing limb contacts the ground, but maximal values differ among the three bones. In detail, the peak force for the contact between the radius and $\mathrm{Cr}$ has a value of $6880 \mathrm{~N}$, the one between the radius and $\mathrm{Ci}$ is $5690 \mathrm{~N}$ and the one between the radius and $\mathrm{Cu}$ is $3050 \mathrm{~N}$ (Fig. 5). These results are consistent with the entry in the curve, where the weight of the horses is carried more in the medial side of the limb than in the lateral 
one. As the limb started the swing phase, contact forces decreases to increase again during maximal flexion of metacarpus without reaching similar values of those obtained during the contact with the ground. Contact points of the first row of carpal bones on the distal radius surface, obtained by tracking the contact forces, are reported. All contact points are present on the radial trochlea at the beginning of the simulation during hyperextension of the metacarpus and then move at approximately $40 \mathrm{msec}$ from the start of the simulation on the palmar aspect of the radius during the flexion of the carpus and then back on medial trochlea during the carpal extension. When macroscopic specimen is analysed, the location of the contact forces obtained from the computational model predicts with good approximation the real points where cartilage damages were observed.

\section{Discussion}

In the current study, we generated a multibody computer model of the entire equine forelimb to derive magnitude and localization of the contact forces at the level of the antebrachio-carpal joint of a horse experiencing a catastrophic fracture during a traditional race. We are interested to depict the interaction between the articular surfaces during motion and describe forces at the instant preceding this catastrophic accident. Differently to the previous study using in silico models, our simulation was based on a real situation rather than a laboratory experiment.

The localization and the magnitude of the contact forces observed at the level of the antebrachio-carpal joint and obtained through computer modelling in our model are comparable to the clinical and pathologic changes observed in the horse examined post-mortem. The maximal contact force derived at the level of the cartilage surface has been localised on the medial styloid process of the radius and occurred at $18 \mathrm{msec}$ from the beginning of the stance phase of the simulated period. We correlated this localization with the consistent inclination of the leading forelimb and the consequent displacement of the horse' centre of mass in relation to the speed of the animal at the entry of the curve, due to the high centripetal force of the animal. The peak of the forces, based on the reconstruction of the entire horse stride in this specific race circuit, occurred probably at the time when the $\mathrm{Cr}$ fractured. The concentration of the force in the centroid of the curvature of medial styloid process matches with the site where the $\mathrm{Cr}$ fractured and, interestingly, the migration of the point of contacts of the forces in that joint matches with the observed pattern of hyaline cartilage injuries.

Fractures of the third carpal bone (C3) and $\mathrm{Cr}$ bones are common in racehorses [9]. Subchondral fragmentation occurs most frequently in the middle carpal joint along the dorso-distal margin of $\mathrm{Cr}$ and the dorso-proximal margin of $\mathrm{C} 3$, followed by the dorso-lateral margin of the distal radius and dorsoproximal margin of $\mathrm{Ci}$, in the antebrachio-carpal joint. The fracture observed in this horse is an unusual fracture in racehorse practice. We have connected the unusual design of the circuit and the small radius of the curves of this track, run at such high speed (the radius of this curve in this circuit is $28.4 \mathrm{mt}$ ) with the forces generated during the accident. At the entry of the curve horses experienced an elevated centripetal acceleration [10] that caused a displacement of their centre of mass, and a tremendous increase of the contact forces in the medial aspect of the antebrachio-carpal joint in the trailing forelimb. 
We estimated a contact peak force of around $7000 \mathrm{~N}$ in a single point between the radial facet and the $\mathrm{Cr}$. This value is underestimated due to the absence of the concurrent forces attributed the flexor and extensor tendons, which were not included in this model.

The values obtained for the contact between the radius and $\mathrm{Cr}$ resulted in line with those obtained in the equine carpal joints using different models $[1,11]$. Literature recognized that the curve in a circuit is a significative factor in the pathogenesis of racehorses injuries $[12,13]$. Kinematics of movement on curved track has been recently investigated in horses at trot [14] and at gallop [10] in standard racetracks, where the radius of the curve ranged from 85 to 200 metres. In horses galloping on a curve, the entire limb and third metacarpal bone inclination increase with speed to counterbalance the increase centripetal acceleration. Usually, horses gallop with the leading limb on the inside of the curve, however the horse we analysed did the opposite as the leading limb, which is the one that sustained the fracture, was outside during the bend. It is possible that this choice could only be the result of the horse's preference [15] or it could be led by the absence of previous training of the horses to race in such a small size track. This factor could also have contributed to the injury, increasing the peak vertical forces sustained by this forelimb. The identification of a severe cartilage breakdown on the radial and intermediate facets of the distal radius could be explained through the analysis of limb loading with the computation modelling. Computation modelling has been previously used to calculate the loads in multiple structures and at multiple sites throughout the distal portion of the third metacarpal bone and the corresponding joint surfaces $[11,16,17]$.

The hypothesis that the horse had previously suffered from severe osteoarthritis of the carpus as a favouring factor was not supported by our data. The microstructural analysis revealed bone densification only in very small areas of the joint, such as the dorsal aspect of the $\mathrm{Ci}$ and on the radial carpal facet of the distal radius. This stage of bone densification is normally observed in adult Thoroughbred, reflecting physiological adaptation of the SCB to intensive training [18]. It is known that the trabecular bone in the carpus is highly responsive to the increased mechanical load of race training [19]. A recent study showed that subchondral bone sclerosis is a result of high-speed exercise [20]. Trabecular thickening and endosteal new bone formation are observed in bones that are subjected to repeated cyclic loading in vivo $[21,22]$. In this horse, the sclerosis of dorsal aspect of the Ci and on the radial carpal facet of the distal radius reflects bone adaptation to training. We have concerns about the ability to develop bone adaptation in the case of traditional city circuit, because the circuit is only experienced during a single annual race and therefore neither adaptation mechanisms are possible. Exposure to this high speed of work and such tight curves is not reproducible under any training conditions. Nevertheless, as the response to the trabecular bone to repetitive loading is anisotropic, the resistance of the bone is higher when the cyclic loading is applied in the direction of the physiological loading angle and lower at other loading angles [23]. Based on current literature, the morphology of the fracture of the Cr was generated under compression, with an high-energy impact at high loading rate [24]. Moreover, the fracture of Mc4 is related to the traction from the lateral collateral ligament of the carpus, which insert to the proximal aspect of the Mc4. One single load at an extreme energy of impact along the non-axis angle has probably overtaken the loading capacity of the cuboid carpal bones in this horse, leading to bone failure in a region 
without any pre-existing subchondral pathology. This consideration is supported by the severity of hyaline cartilage lesions which does not matched with normal microstructure observed in the SCB, indicative of an acute rather than a bone fatigue injury. In fact, the lesions are not uniformly distributed on the distal radius, but interestingly, they are localized in the points where the model predicts the higher forces of contact. Neither histological characteristics of the chronicity of the lesions, like the presence of chondrocytes cluster and the reduction of chondrocytes density were detected at histopathology. Bone geometry, bone material properties, load direction and the speed of application are the main factor involved in bone failure [25]. The cuboid bones in the lateral aspect of the carpus allow greater dissipation of the axial loading more easily thanks to the arrangement of the fourth carpal bone (C4) between the $\mathrm{Cu}$ and the $\mathrm{Ci}$ and the similar arrangement of the $\mathrm{Ci}$ between the $\mathrm{C} 4$ and the third carpal bone (C3) $[26,27]$. This geometric arrangement of the bones favours the horizontal translation of the bones and their recoil, due to the strong intercarpal ligaments. This type of mechanism is impossible in the medial aspect of the joint, where this horse experienced the fracture.

Our in silico model presents some limitations; first of all not all the points where macroscopic lesions were found were completely explained by the model. This could be probably due to the necessary simplifications introduced in the model respect to the in vivo situation. In addition, the model was retrospectively created and refers to a single case with an unusual injury. The reason why kinematics data were not imported in the model is, as mentioned before, that there is no possibility to simulate the same track design, ground condition and settings under training for ethical reason.

\section{Conclusion}

Inverse dynamic model of a horse galloping during a city circuit competition has been able to predict catastrophic injury occurred to the horse in the present study. In term of prevention of this type of injury, this study supports the consideration that some city tracks should be redesigned to increase their radius of curvature to accommodate horses running at high speed. Prior to make a circuit, the forces produced during the race on the basis of the circuit design could be assessed using a similar model, to offer a safer racing environment and preserve horses' welfare.

\section{Methods}

Tissues were harvested from a 7-years old Thoroughbred (TB), which was euthanized as consequence of a catastrophic fracture, which took place during the final competition of a traditional horse race ("Palio di Asti", Italy) in 2015. The injury occurred on the track realized in Piazza Alfieri, the largest square in the city centre of Asti. The racing circuit has triangular shape and the race is performed in a clockwise direction. The horse of the study fell down in correspondence of the entrance of the second curve due to sudden collapse of the left forelimb (LF) during the stance phase of the stride, as observed on the recorded tape of the competition. The animal exhibited a left leading transverse gallop. After standing up, the horse was not weight bearing on the LF and the carpus was barely unstable. First aid was immediately provided including immobilisation of the limb and the animal was transported to the closest equine hospital. After 
admission, radiographic examination of the entire LF was performed and $\mathrm{Cr}$ bone fracture was diagnosed. The owner denied any treatment and therefore the horse was humanely euthanatized. After obtaining the owner consent, anatomical specimens were collected for study. The entire flow chart of the study is reported in Figure 6.

\section{CT scan}

Limb was amputated at the level of the proximal epiphysis of the radius and immediately frozen. Afterwards CT scan (General Electric Hi-Speed Fx; detector rows of $2.0 \mathrm{~mm}$ each; collimation, $120 \mathrm{kVp}$; tube charge, $130 \mathrm{mAs}$; and pitch, 1.0) of the limb was performed to evaluate the configuration of the fracture lines. The specimen was placed with the carpus in full extension on the CT table into a legsupporting pad with the long axis of the limb parallel to the CT gantry. Contiguous $2.0 \mathrm{~mm}$ transverse slices were acquired of the region of interest (ROI), from the diaphysis of the radius to the middle third of the third metacarpal region. Specimen was examined in a proximal-to-distal direction along the transverse, sagittal and coronal cutting plans, and the generated images were viewed using a bone window. Elaboration in 2D and volume rendering of the bony structures was performed using a DICOM software (OsiriX Lite; Pixmeo SARL, Bernex, Switzerland) and evaluated by an experience radiologist.

\section{Pathology}

Macroscopic evaluation of the antebrachio-carpal and inter-carpal joints was performed after careful dissection and disarticulation of the carpal joints compartments. Macroscopic cartilage lesions were scored accordingly with the technique described by Mcllwraith et al.[28]. Indian ink solution diluted with phosphate-buffered saline (1:5) was used to stain the superficial hyaline cartilage as described by

Schmitz et al. [29] and the stained specimens were photographed using a high-resolution digital camera.

Findings were evaluated according to the following classification: grade 1 (intact surface), when surface was normal and smooth in appearance and did not retain Indian ink, grade 2 (minimal fibrillation), when surface retained Indian ink as elongated specks or light-grey patches, grade 3 (overt fibrillation), when area were velvety in appearance and retained Indian ink as intense black patches, and grade 4 (erosion), when area of cartilage exposing the underlying subchondral bone was evident.

\section{Further Specimen processing}

The distal epiphysis of the radius, the proximal and the distal rows of the cuboid carpal bones with the corresponding articular surfaces were carefully dissected and stored at $-20^{\circ} \mathrm{C}$, until further examination. All disarticulated bones were identified with an ID number.

\section{Micro-computed tomographic $(\mu C T)$ analysis}


Specimens were submitted to the Istututo Ortopedico Rizzoli (IOR, Bologna, Italy) for $\mu C T$ scan (highresolution $\mu$ CT Skyscan 1176 Bruker, Belgium). During $\mu C T$ acquisition a $65-k V$ voltage and a current of $385 \mu \mathrm{A}$ were applied to the source interposing a $1 \mathrm{~mm}$ thick aluminium filter. Specimens were rotated $180^{\circ}$ following a $0.4^{\circ}$ rotation steps. The scan images obtained ( $1336 \times 3936$ pixels) have a nominal resolution (pixel size) of $17.5 \mu \mathrm{m}$; and were then reconstructed with the NRecon program (version 1.6.10.4, Bruker) to obtain 5911 micro-tomographic sections (each $3936 \times 3936$ pixels, keeping the relative pixel size). As correction factors for the reconstructions, in addition to the specific alignment relative to each single scan, beam hardening correction, smoothing and ring artefacts reduction were used. Specimens were examined along the transverse, sagittal, and coronal cutting plans. Further elaborations in $2 \mathrm{D}$ and volume rendering were performed. False colours were attributed to tissues in relation to their structural density using yellow ochre for the bone and light blue for the hyaline cartilage. Multiple planes for bone assessment were planned in the distal radial epiphysis, where the majority of the cartilage lesions were localised, to map the entire subchondral bone in relation to the severity of hyaline cartilage lesions. The $2 \mathrm{D}$ sections were examined to identify a pattern of bone degenerative changes using a structural score, where score 1: no irregularities, score 2: surface irregularities and pits sometimes associated with focal porosity in the subchondral bone, score 3: surface irregularities and pits with marked focal porosity throughout the full depth of the subchondral and trabecular bone[4]. Discrete volumes of interest ( $\mathrm{VOI}$ ) in the distal radial epiphysis and in the corresponding cuboid carpal bones were identified for quantitative analyses. Parameters measured included bone volume fraction (BV/TV), trabecular thickness (Tb.Th), trabecular separation (Tb.Sp) and number (Tb.N), trabecular pattern factor (Tb.Pf), structure model index (SMI), degree of anisotropy (Da) and C.Th. Bone mineral density was not considered for the analysis because the potential bias related to the freezing processing of the specimen. After $\mu \mathrm{CT}$ assessment, specimens were stored again in PBS at $+4^{\circ} \mathrm{C}$.

\section{Histopathology}

Specimens of distal radial epiphysis were processed for histological analysis. Multiple cut lines were planned in the articular surface of the distal radius to map the entire articular cartilage of this specimen in relation of the severity of the macroscopic evaluation. Fourteen osteochondral pieces were obtained (Figure 1). Cuts for histological examination were preferentially performed in the regions where macroscopic cartilage lesions were observed. After fixation in $10 \%$ formalin for $24 \mathrm{~h}$ and rinsing in water, specimens were decalcified with a $5 \%$ formic acid solution and $4 \%$ hydrochloric acid in $1 \mathrm{~L}$ distilled water, respecting the proportion for the desired volume and remained in a descaling solution for one month, and the hardness of the bone component was systematically checked. After descaling, samples were rinsed in water and then dehydrated in an increasing series of ethyl alcohol (70\%, 95\% and $100 \%)$, passed in xylol and included in paraffin blocks. For each osteochondral piece, two sections of $5 \mu \mathrm{ms}$ thickness were cut along the transverse plane of the carpus to observe both the hyaline cartilage and the corresponding SCB. Sections were stained with H\&E and with SOFG. The stained sections were then acquired at 100x for further analysis through a digital scanner (Leica Biosystems) and assessed by one author ( $A B$ ) using the OARSI osteoarthritis cartilage histopathology assessment system [28] to determine the histopathology 
grading of the articular damage. A site-by-site comparison was performed between the histopathology evaluation of the hyaline cartilage and the corresponding $\mu \mathrm{CT}$ sections of the SCB.

\section{Computation modelling}

Starting from the CT scans all the articulating bones of the carpal joint were segmented and the respective 3D solid geometries were created using commercial software for 3D image processing (Mimics, Materialize, USA). Specifically, since the $\mathrm{Cr}$ was fractured into two main pieces, it was virtually reconstructed by aligning the bone fragments and applying a wrapping function to reproduce the nonfractured bone surface. Both the segmentation and reconstruction processes were supported by a veterinary surgeon $(A B)$. Therefore, the obtained $3 D$ geometries were imported into a multibody software (MD Adams, MSC Software Corporation, USA), where a dynamic model was implemented.

The model included the following bones obtained from the CT scans (Figure 7): the distal radius, the $\mathrm{Cr}$, the $\mathrm{Ci}$, the the $\mathrm{Cu}$, the second carpal bone (C2), the $\mathrm{C} 3$, the $\mathrm{C} 4$, the proximal metacarpal bone. In addition to the mentioned bones, the humerus, the proximal radius, the ulna, the distal metacarpal bone, and the phalanges were included (Figure 8a) by adapting standardized geometries to the specific horse sizes. A value of density equal to $1590 \mathrm{~kg} / \mathrm{m}^{3}$ was assigned to the bones [30], thus, the inertial characteristics and centre of mass were computed for each body segment. Moreover, the body of the horse was simplified by using a cylindrical geometry having a mass equal to the body weight, that is $500 \mathrm{~kg}$.

As regarding the articular joints, the shoulder, elbow, and fetlock were modelled as hinges, thus, each of them allows for only a rotation (i.e., 1 degree of freedom) on the sagittal anatomical plane, whereas the articulations between phalanges were considered fixed (Figure $\mathbf{8 b}$ ). Since this study was focused on the investigation of the forces generated inside the carpal joint, contacts between the articulating surfaces of the carpal bones and the retaining actions of the articular ligaments were implemented. However, the complexity of the joint, due to the numerosity of its anatomical structures (i.e., the various osseous segments, ligaments, and tendinous structures), required the introduction of some modelling simplifications. First, the relative motion among the $\mathrm{C} 2, \mathrm{C} 3$ and $\mathrm{C} 4$ bones was neglected by fixing them together. Second, only the collateral ligaments and the dorsal retinaculum were modelled whereas the constraining effect of the intercarpal ligaments and soft tissues that surround the joint was represented by means of bushing elements. Specifically, each collateral ligament was represented by means of two branches, i.e., the long and short medial collateral ligament (I-MCL and s-MCL), the long and short lateral collateral ligament (I-LCL and s-LCL). The dorsal retinaculum was represented by two branches per joint side, i.e., the long and short medial retinaculum (I-MR and s-MR), the long and short lateral retinaculum (ILR and s-LR). Each branch was modelled as a non-linear only-tension spring [31] having the characteristic stiffness parameters adapted from the literature [30] and the slack lengths of the ligaments tuned, by means of preliminary simulations, starting from the modelled ligament lengths measured with the joint at its resting position (Additional file 2). Origin and insertion points of the branches were deduced on the base of anatomical references (Figure 7b-d). Each bushing element was implemented to generate a six- 
components force (i.e., 3 translational forces and 3 torques) acting between two bones proportionally to their relative linear and angular displacements and velocities (Addition file 3).

The intra-articular contact forces were calculated by means of an interpenetration function taking into account the total C.Th between the contacting bones (Additional file 4). The value of the C.Th of the carpal bones and radius considered in the study has been previously reported in literature by Murray Rachel et al. [32] and Lee et al. [33]. Therefore, three different contact pairs were defined between the distal radius and the $\mathrm{Cr}, \mathrm{Ci}, \mathrm{Cu}$ bones, respectively. Articular contacts were assumed frictionless. In addition, a further contact was defined between the limb and the ground. Also, the gravity force was considered for the simulation.

To simulate the full-gallop of the horse when the accident occurred, averaged kinematic curves found in literature [34], expressed as flexion/extension angle vs. percentage of stride, were properly scaled in time and prescribed to the joint represented in the model.

Furthermore, video footages of the race, acquired from different points of view, were analysed to extract useful information to replicate the dynamic conditions of the accident, such as the horse velocity and the front limb inclination with respect to the ground (Figure 8a), i.e., $14 \mathrm{~m} / \mathrm{s}$ and $56^{\circ}$, respectively. The whole simulated stride has a duration of $0.102 \mathrm{msec}$.

Locations and magnitudes of the intra-articular contact forces were obtained as output from the computational simulation and compared with the location of the cartilage damage observed postmortem.

\section{Abbreviations}

SBC: Subchondral bone; Cr: radial carpal bone; Ci: intermediate carpal bone; Mc4:fourth metacarpal bone; LF: left forelimb; Cu: ulnar carpal bone; C.Th: cartilage thickness; H \& E: hematoxylin and eosin; SOFG: safranin-0 fast green; C2: second carpal bone; C3: third carpal bone; C4: fourth carpal bone; TB:

Thoroughbred; CT: computed tomography; $\boldsymbol{\mu C T}$ : micro computed tomography; 2D: two-dimensional; 3D: three-dimensional; I-MC: long medial collateral ligament; s-MCL: short collateral ligament; I-LCL long lateral collateral ligament; s-LCL: short lateral collateral ligament; I-MR: long medial retinaculum; s-MR: short medial retinaculum; I-LR: long lateral retinaculum; s-LR: short lateral retinaculum.

\section{Declarations}

\section{Ethics approval and consent to participate:}

Informed consent was obtained from owner of the horse.

\section{Consent for publication:}




\section{Availability of data and materials:}

All data generated or analysed during the study are included in this specific article and available from the corresponding author on reasonable request.

\section{Competing interests:}

The authors declare that they have no competing interests

\section{Funding:}

none

\section{Authors' contributions:}

$E P, A P$ and $A B$ conceived and design the study. AV, APP, MS and MF were responsible for acquisition and extraction of data. MT, GP and EMZ designed, projected and developed the model. EP, FC and AB wrote the manuscript; $E P$ and $A B$ interpreted the results and critically revised the manuscript. All authors have read and approved the manuscript.

\section{Acknowledgements:}

not applicable

\section{References}

1. Palmer JL, Bertone AL, Litsky AS. Contact area and pressure distribution changes of the equine third carpal bone during loading. Equine Veterinary Journal. 1994;26:197-202.

2. Wong $M$, Carter DR. Articular cartilage functional histomorphology and mechanobiology: a research perspective. Bone. 2003;33:1-13.

3. Stolberg-Stolberg JA, Furman BD, Garrigues NW, Lee J, Pisetsky DS, Stearns NA, et al. Effects of cartilage impact with and without fracture on chondrocyte viability and the release of inflammatory markers. Journal of Orthopaedic Research. 2013;31:1283-92.

4. Lacourt M, Gao C, Li A, Girard C, Beauchamp G, Henderson JE, et al. Relationship between cartilage and subchondral bone lesions in repetitive impact trauma-induced equine osteoarthritis. Osteoarthritis and Cartilage. 2012;20:572-83. 
5. Muir P, Peterson AL, Sample SJ, Scollay MC, Markel MD, Kalscheur VL. Exercise-induced metacarpophalangeal joint adaptation in the Thoroughbred racehorse. Journal of Anatomy. 2008;213:706-17.

6. Bertuglia A, Lacourt M, Girard C, Beauchamp G, Richard H, Laverty S. Osteoclasts are recruited to the subchondral bone in naturally occurring post-traumatic equine carpal osteoarthritis and may contribute to cartilage degradation. Osteoarthritis and Cartilage. 2016;24:555-66.

7. Putame G, Terzini M, Bignardi C, Beale B, Hulse D, Zanetti E, et al. Surgical Treatments for Canine Anterior Cruciate Ligament Rupture: Assessing Functional Recovery Through Multibody Comparative Analysis. Front Bioeng Biotechnol. 2019;7. doi:10.3389/fbioe.2019.00180.

8. Terzini M, Zanetti EM, Audenino AL, Putame G, Gastaldi L, Pastorelli S, et al. Multibody modelling of ligamentous and bony stabilizers in the human elbow. Muscles Ligaments Tendons J. 2018;7:493502.

9. Pd R, R H, Nj D, K O. Epidemiological study of wastage among racehorses 1982 and 1983. Vet Rec. 1985;116:66-9.

10. Parkes RSV, Pfau T, Weller R, Witte TH. The effect of curve running on distal limb kinematics in the Thoroughbred racehorse. PLOS ONE. 2020;15:e0244105.

11. Harrison SM, Whitton RC, Kawcak CE, Stover SM, Pandy MG. Relationship between muscle forces, joint loading and utilization of elastic strain energy in equine locomotion. J Exp Biol. 2010;213 Pt 23:3998-4009.

12. Parkin TDH, Clegg PD, French NP, Proudman CJ, Riggs CM, Singer ER, et al. Catastrophic fracture of the lateral condyle of the third metacarpus/metatarsus in UK racehorses - fracture descriptions and pre-existing pathology. The Veterinary Journal. 2006;171:157-65.

13. Ueda Y, Yoshida K, Oikawa M. Analyses of race accident conditions through use of patrol video. Journal of Equine Veterinary Science. 1993;13:707-10.

14. Kallerud AS, Hernlund E, Byström A, Persson-Sjodin E, Rhodin M, Hendrickson EHS, et al. Non-banked curved tracks influence movement symmetry in two-year-old Standardbred trotters. Equine Vet J. 2020.

15. Williams DE, Norris BJ. Laterality in stride pattern preferences in racehorses. Animal Behaviour. 2007;74:941-50.

16. Malekipour F, Whitton RC, Lee PV-S. Distribution of mechanical strain in equine distal metacarpal subchondral bone: A microCT-based finite element model. Medicine in Novel Technology and Devices. 2020;6:100036.

17. Merritt JS, Pandy MG, Brown NAT, Burvill CR, Kawcak CE, Mcllwraith CW, et al. Mechanical loading of the distal end of the third metacarpal bone in horses during walking and trotting. Am J Vet Res. 2010;71:508-14.

18. Kawcak CE, Mcllwraith CW, Norrdin RW, Park RD, Steyn PS. Clinical effects of exercise on subchondral bone of carpal and metacarpophalangeal joints in horses. Am J Vet Res. 2000;61:1252-8. 
19. Tidswell HK, Innes JF, Avery NC, Clegg PD, Barr ARS, Vaughan-Thomas A, et al. High-intensity exercise induces structural, compositional and metabolic changes in cuboidal bones - findings from an equine athlete model. Bone. 2008;43:724-33.

20. Martig S, Hitchens PL, Lee PVS, Whitton RC. The relationship between microstructure, stiffness and compressive fatigue life of equine subchondral bone. Journal of the Mechanical Behavior of Biomedical Materials. 2020;101:103439.

21. Lynch ME, Main RP, Xu Q, Schmicker TL, Schaffler MB, Wright TM, et al. Tibial compression is anabolic in the adult mouse skeleton despite reduced responsiveness with aging. Bone. 2011;49:439-46.

22. Radin EL, Parker HG, Pugh JW, Steinberg RS, Paul IL, Rose RM. Response of joints to impact loading - III: Relationship between trabecular microfractures and cartilage degeneration. Journal of Biomechanics. 1973;6:51-7.

23. Dendorfer S, Maier HJ, Taylor D, Hammer J. Anisotropy of the fatigue behaviour of cancellous bone. Journal of Biomechanics. 2008;41:636-41.

24. Browner BD. Skeletal Trauma: Basic Science, Management, and Reconstruction. Elsevier Health Sciences; 2009.

25. DeGoede KM, Ashton-Miller JA, Schultz AB. Fall-related upper body injuries in the older adult: a review of the biomechanical issues. Journal of Biomechanics. 2003;36:1043-53.

26. Bramlage LR, Schneider RK, Gabel AA. A clinical perspective on lameness originating in the carpus. Equine Veterinary Journal. 1988;20:12-8.

27. Deane NJ, Davies AS. The function of the equine carpal joint: A review. New Zealand Veterinary Journal. 1995;43:45-7.

28. Mcllwraith CW, Frisbie DD, Kawcak CE, Fuller CJ, Hurtig M, Cruz A. The OARSI histopathology initiative - recommendations for histological assessments of osteoarthritis in the horse. Osteoarthritis and Cartilage. 2010;18:S93-105.

29. Schmitz N, Laverty S, Kraus VB, Aigner T. Basic methods in histopathology of joint tissues. Osteoarthritis and Cartilage. 2010;18:S113-6.

30. Swanstrom MD, Zarucco L, Hubbard M, Stover SM, Hawkins DA. Musculoskeletal Modeling and Dynamic Simulation of the Thoroughbred Equine Forelimb During Stance Phase of the Gallop. Journal of Biomechanical Engineering. 2004;127:318-28.

31. Blankevoort L, Kuiper JH, Huiskes R, Grootenboer HJ. Articular contact in a three-dimensional model of the knee. Journal of Biomechanics. 1991;24:1019-31.

32. Rachel CM, DeBowes RM, Gaughan EM, Mosier DE, Athanasiou KA. Variations in the Biomechanical Properties of Articular Cartilage of the Midcarpal Joint of Normal Horses. Vet Comp Orthop Traumatol. 1995;08:133-40.

33. Lee H, Kirkland WG, Whitmore RN, Theis KM, Young HE, Richardson AJ, et al. Comparison of equine articular cartilage thickness in various joints. Connective Tissue Research. 2014;55:339-47. 
34. Back W, Schamhardt HC, Barneveld A. Kinematic comparison of the leading and trailing fore- and hindlimbs at the canter. Equine Veterinary Journal. 1997;29:80-3.

\section{Figures}
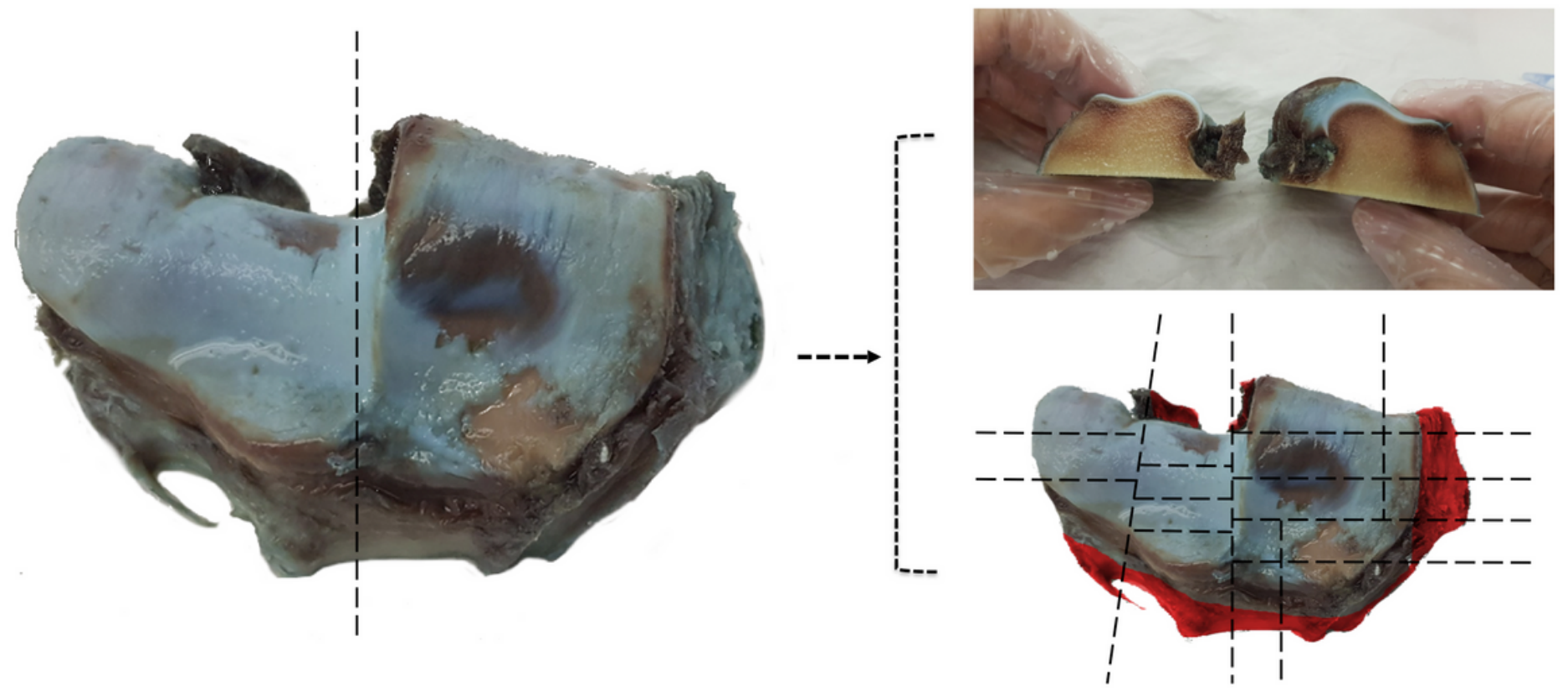

\section{Figure 1}

Macroscopic aspect of the articular surface of the distal radius after dissection of the antebrachio-carpal joint, after deposition of Indian ink. Hyaline articular cartilage is severely damaged, as shown after Indiank ink staining. Planned multiple cut-lines in the articular surface of the antebrachiocarpal joint are indicated to perform histological examination of the entire hyaline articular cartilage 


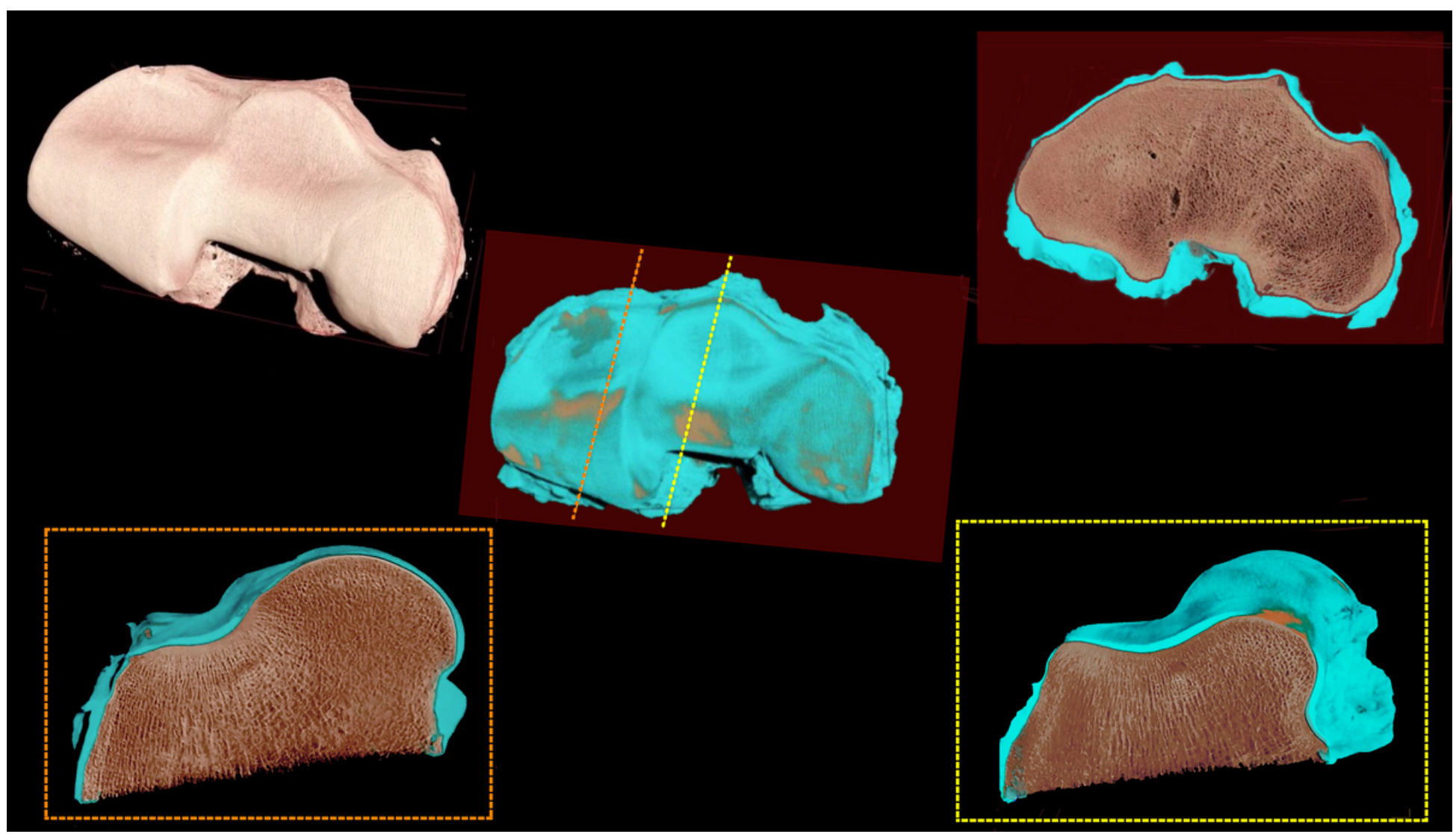

Figure 2

$\mu \mathrm{CT}$ scan with the corresponding volume rendering of the entire distal radius (antebrachio-carpal joint). The sagittal, coronal and transverse plans of the distal radial epiphysis are shown. Plane of cuts are indicated with the corresponding colours 


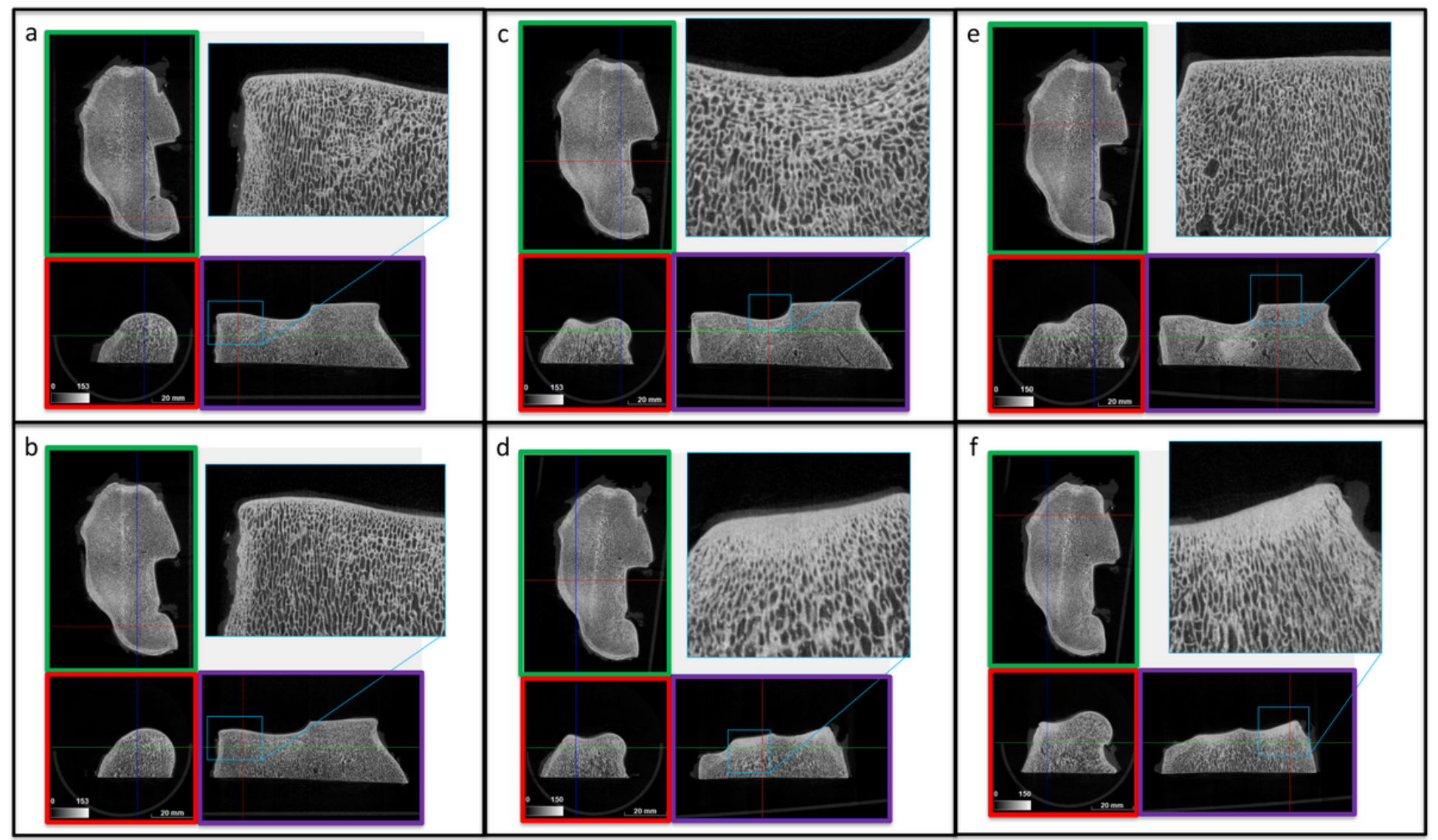

Figure 3

Qualitative $\mu C T$ evaluation at the level of the radial, intermediate and ulnar facets of the distal radial epiphysis. The panels represent different sections from medial to lateral and from dorsal to palmar of the distal radial epiphysis. Six subpanels are presented. (a-b) Ulnar facet. (c-d) Intermediate facet. (e-f) Radial facet. Each subpanel includes four images: the transverse plan (green frame), the sagittal plan (red frame), the coronal plan (violet frame), and the magnified area indicated with the blue frame in the coronal plans. An area with moderate densification of the subchondral bone were identified in $d$ and $f$ (blue frames), in the radial and intermediate facets of the distal radius, while in the other panels the architecture of the trabecular bone is normal. 

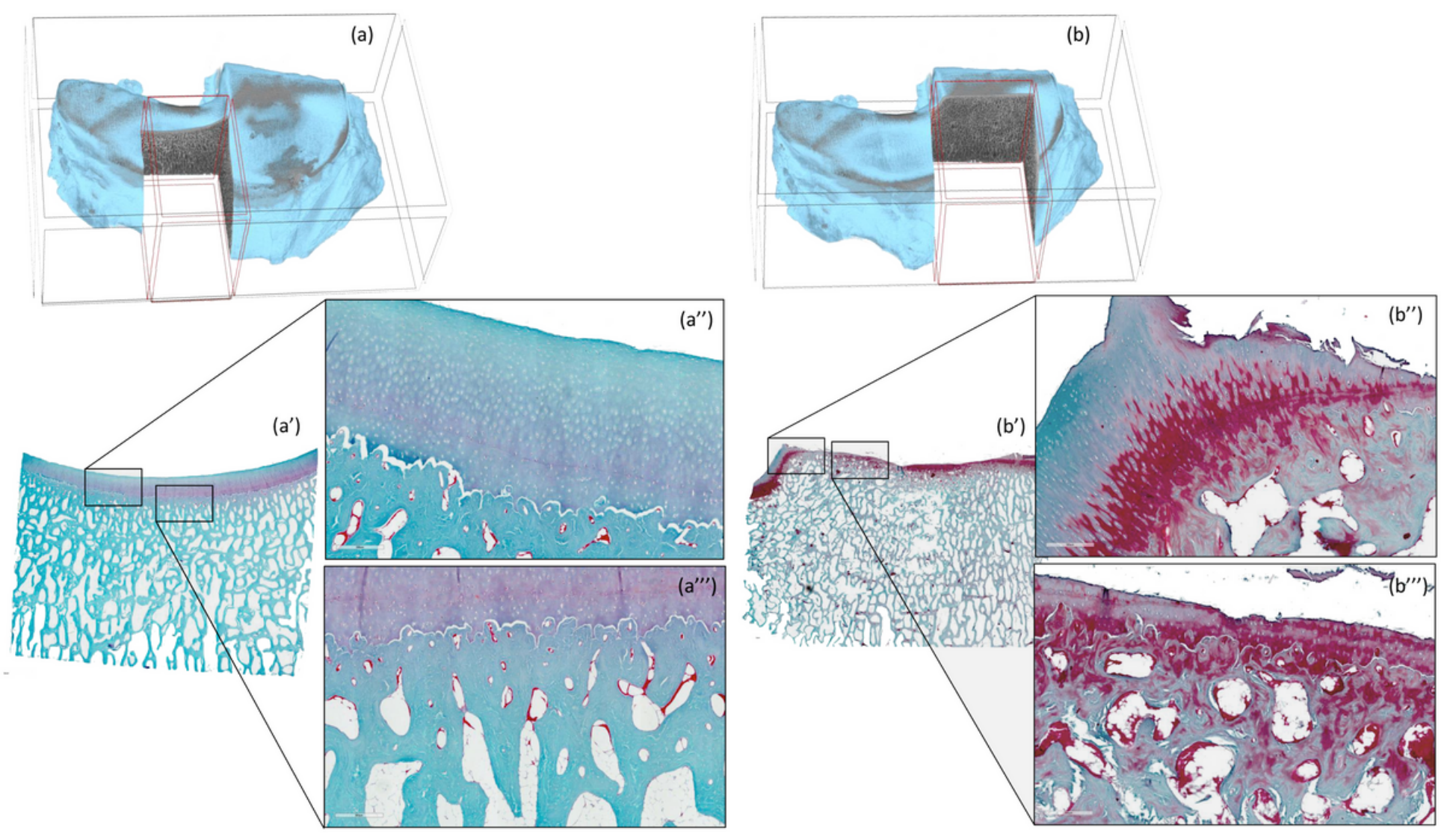

\section{Figure 4}

Histopathology specimens of the hyaline cartilage and subchondral bone at the level of the intermediate $\left(a, a^{\prime}, a^{\prime \prime}\right.$ and $\left.a^{\prime \prime \prime}\right)$ and radial ( $b, b^{\prime}, b^{\prime \prime}$, and b') facets of the articular surface of the radius. Particulars of the intermediate facet ( $a$ " and $\left.a^{\prime \prime \prime}\right)$, with normal hyaline cartilage. Particulars of the radial facet ( $b^{\prime \prime}$ and $\left.b^{\prime \prime \prime}\right)$, with extensive loss of the hyaline cartilage. The cartilage appeared extensively torn more than degenerated. 

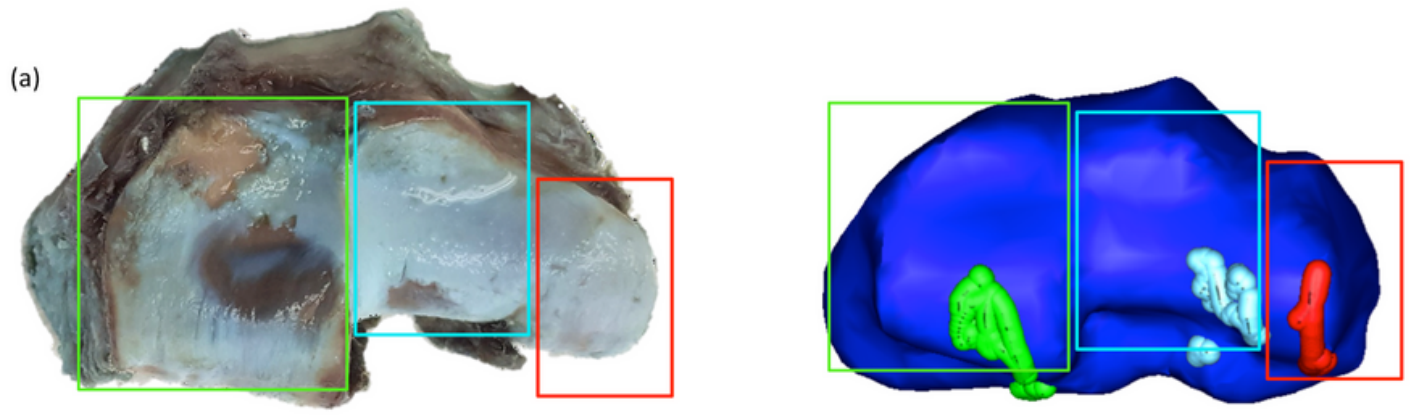

(b)

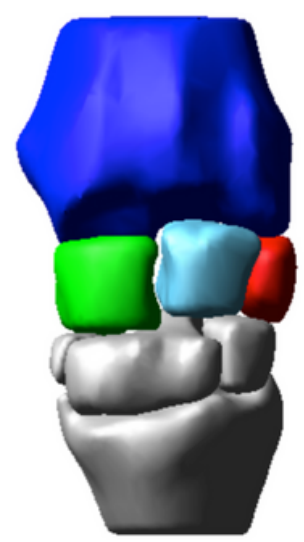

(c)

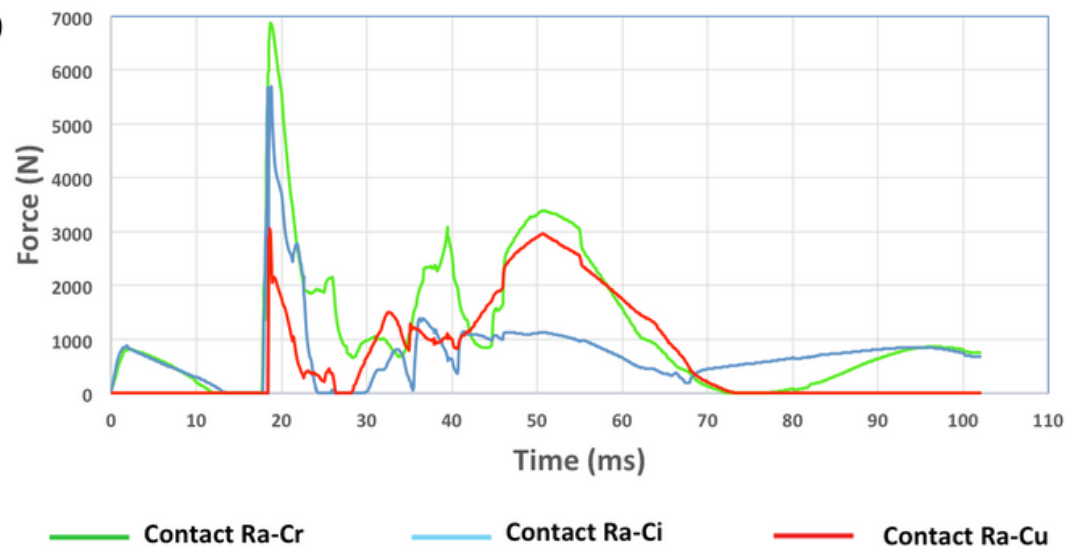

\section{Figure 5}

(a) Comparison between cartilage damage on the specimen (left) and the contact locations tracked resulting from the simulation; (b) 3D model detail of the carpal joint showing the contacting bones with the radius in blue, the radial carpal bone $(\mathrm{Cr})$ in green, the intermediate carpal bone $(\mathrm{Ci})$ in light blue and the ulnar carpal bone $(\mathrm{Cu})$ in red; (c) Plot showing the magnitude of the contact forces between the radius $(\mathrm{Ra})$ and the $\mathrm{Cr}$ in green, the $\mathrm{Ci}$ in light blue and the $\mathrm{Cu}$ in red, respectively. 


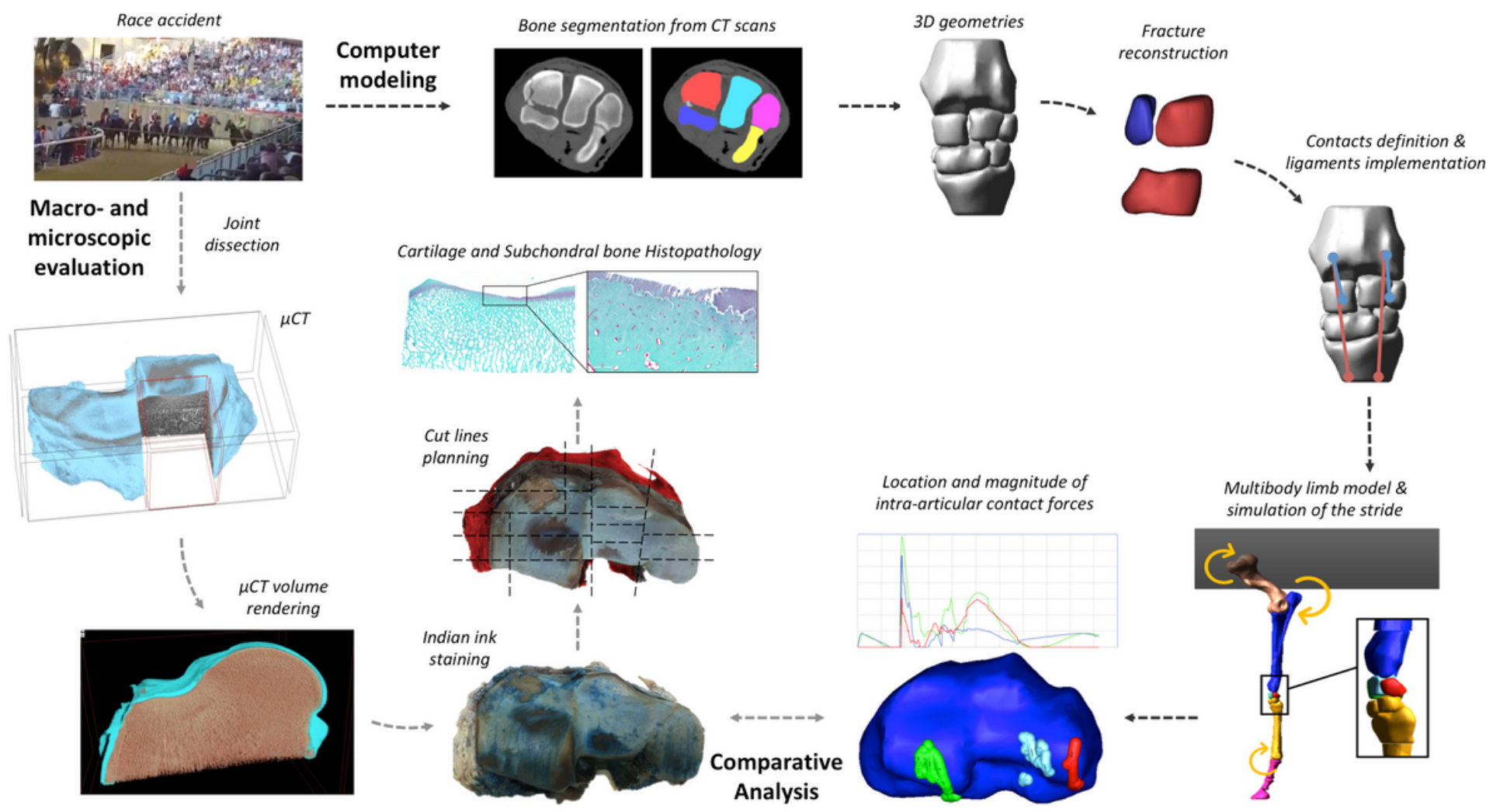

Figure 6

Schematic flow-chart of the study

(a)

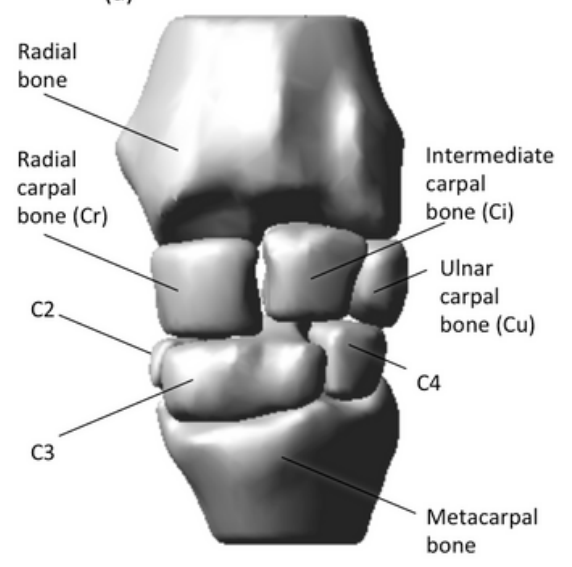

(b)

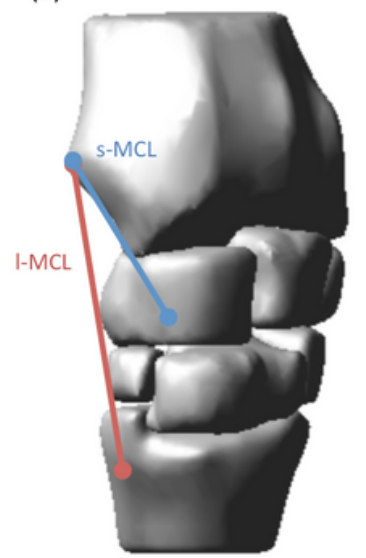

(c)

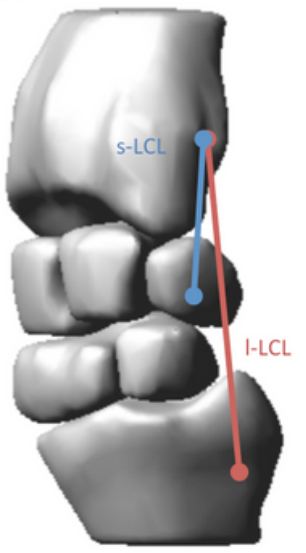

(d)

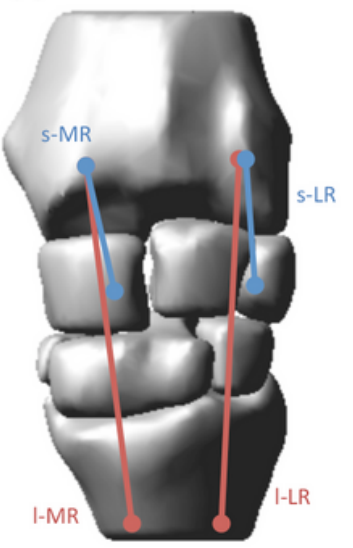




\section{Figure 7}

(a) Frontal view of the 3D recostruction of the carpal joint obtained from the CT scans with all bone segments considered; (b) medial showing the short (s-MCL) and long (I-MCL) brunches of the medial collateral ligament; (c) lateral view showing the short (s-LCL) and long (I-LCL) brunches of the lateral collateral ligament; (d) frontal view showing the short (s-MR) and long (I-MR) brunches of the medial retinaculum and the short (s-LR) and long (I-LR) brunches of the lateral retinaculum.

(a)

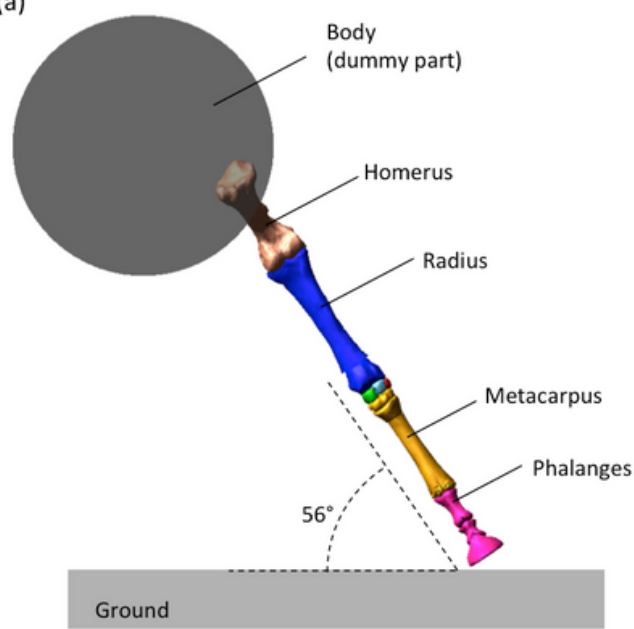

(b)

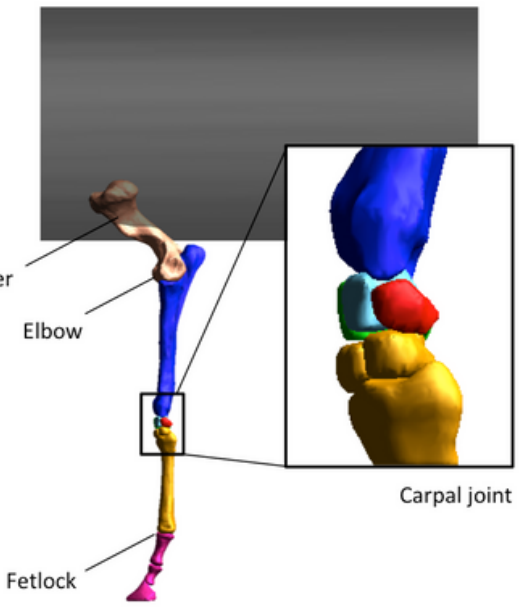

\section{Figure 8}

Frontal view (a) of the model with indicated the inclination angle between ground and limb; sagittal view (b) of the model with detailed windows on the carpal joint.

\section{Supplementary Files}

This is a list of supplementary files associated with this preprint. Click to download.

- additionalfile1.mov

- additionalfile2Springelementimplementation.docx

- Additionalfile3Bushingelementimplementation.docx

- Additionalfile4Contactimplementation.docx 